\title{
A METHOD OF GENERATING PANORAMIC STREET STRIP IMAGE MAP WITH MOBILE MAPPING SYSTEM
}

\author{
Chen Tianen ${ }^{\text {a }}$, Kohei Yamamoto ${ }^{\text {a }}$, Kikuo Tachibana ${ }^{\text {a }}$ \\ a PASCO CORP. R\&D CENTER, 2-8-10 Higashiyama Meguro-Ku, Tokyo 153-0043, JAPAN - (tnieah3292, kootho1810, \\ kainka9209)@pasco.co.jp
}

Commission I, ICWG I/VA

KEY WORDS: Mobile Mapping System, Omni-Directional Camera, Laser Point Cloud, Street-Side Map, Image Stitching

\begin{abstract}
:
This paper explores a method of generating panoramic street strip image map which is called as "Pano-Street" here and contains both sides, ground surface and overhead part of a street with a sequence of $360^{\circ}$ panoramic images captured with Point Grey's Ladybug3 mounted on the top of Mitsubishi MMS-X 220 at 2m intervals along the streets in urban environment. On-board GPS/IMU, speedometer and post sequence image analysis technology such as bundle adjustment provided much more accuracy level position and attitude data for these panoramic images, and laser data. The principle for generating panoramic street strip image map is similar to that of the traditional aero ortho-images. A special 3D DEM(3D-Mesh called here) was firstly generated with laser data, the depth map generated from dense image matching with the sequence of $360^{\circ}$ panoramic images, or the existing GIS spatial data along the MMS trajectory, then all $360^{\circ}$ panoramic images were projected and stitched on the 3D-Mesh with the position and attitude data. This makes it possible to make large scale panoramic street strip image maps for most types of cities, and provides another kind of street view way to view the $360^{\circ}$ scene along the street by avoiding the switch of image bubbles like Google Street View and Bing Maps Streetside.
\end{abstract}

\section{INTRODUCTION}

Much more efforts have been invested into large scale urban 3D model construction from vision-based 3D modelling (Baillard et al., 1999; El-Hakim, 2002; Bentrah et al., 2004a; Surendra et al., 2014) and fusion 3D laser point clouds with images (Frueh et al., 2005; Zhao and Shibasaki, 2001; Stamos and Allen, 2000) for these applications such as navigation, driving direction pre-visualizations and augmented reality as demonstrated in Google Earth or Microsoft Virtual Earth in the last decades, but the progress on the street view level is not so remarkable and the achieved results are still on the level of research. The reasons may be that the ground environments of the urban streets are tens of times much more complex, and often lack textured areas, contain repetitive structures, moving cars and pedestrians, many occlusions, strong lighting changes, and cast shadows. These properties make the above two methods difficult in the sense of finding enough reliable point matches between overlapping images and extracting key skeleton points from 3D laser point clouds for following 3D model construction. To create visually pleasing modes at a high level of detail in urban environments, an enormous amount of manual work, such as importing the geometry obtained from construction plans, polylines, points or selecting primitive shapes and correspondence points for image-based modelling or complex data acquisition are involved. In fact, like aero ortho-images in GIS, a panoramic street strip image map is also a low-cost, shortcut and useful GIS data to meet the basic requirements of the above applications and these applications such as virtual travel and architectural walkthroughs to some extent. It could be also suitable for registration, transmission, visualization of street landscapes, and displayed on the internet to provide users a visual seamless immersive summary of a city.

Two kinds of low-cost, shortcut and functionary methods to provide users much more detail immersive street scenes are systems such as Google Street View and Microsoft Live StreetSide that enable users to virtually visit cities by navigating between immersive $360^{\circ}$ panoramic images (Vincent 2007), or bubbles, and multi-perspective strip panoramas that can provide a visual summary of a city street with a long image strip constructed a sequence video images along the street (Román et al. 2004; Román and Lensch 2006; Agarwala et al. 2006; Rav-Acha et al. 2008).

The former could provide a photorealistic impression from a particular viewpoint inside a bubble by panning and zooming the bubble or the image, but they do not provide a good visual sense of a larger aggregate such as a whole city block or longer street and need vast amount of geo-registered panoramic imagery. Navigating such photo collections is laborious and similar to hunting for a given location on foot: walk "along" the street, (e.g., jumping from bubble to bubble in Street View) looking around, until you find the location of interest. Since automatically geo-located addresses and/or GPS readings are often off by several meters or more especially in urban settings, visually searching for a location is often needed. Within a bubble, severe foreshortening of a street side from such a distance makes recognition almost impossible. Furthermore, a huge data size is inevitable to archive an entire city.

The latter could provide a useful visual summary of all the landmarks along a city street using a long image strip

* Corresponding author 
generated with slit or line scanning mechanism (Zheng and Tsuji, 1992; Gupta and Hartley, 1997) and image patch stiching or video mosaicking mechanism (Zheng et al., 2004; Zhu et al., 2004). Kopf et al. (2010) provided a street slide which dynamically combines the aspects of the immersive nature of bubbles and the overview properties of the multiperspective strip panoramas. As the user slides sideways, the multi-perspective panorama is dynamically constructed and rendered to simulate either a pseudo perspective or hyperperspective view of the street side from a distance by aligning and overlaying perspective projections of dropped resolution bubble images oriented towards the street side. Unfortunately, because all of the source imagery is compressed into a single flat summary, they lack the spatial geographic information as the traditional ortho-images generated from aero and satellite images.
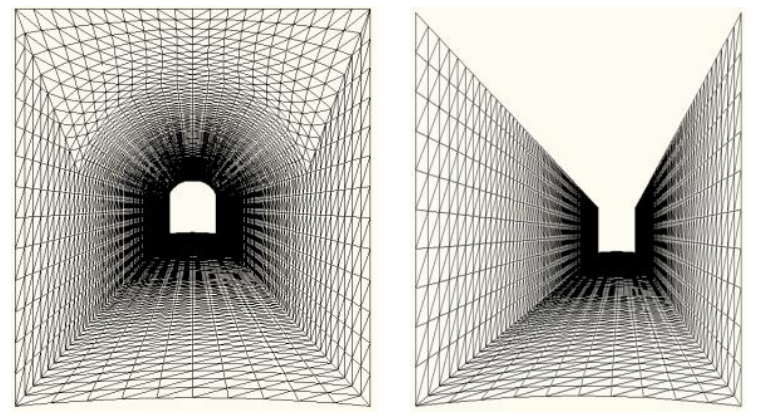

(a) Caged 3D Mesh

(b) Trough-shaped 3D Mesh

Figure 1. Two Types of 3D Meshes Used as Projection Base Plans for Panoramic Street Strip Image Map Generation.

This paper explores a method of making panoramic street strip image map which is called as "Pano-Street" here and contains both sides, ground surface and overhead part all directions of a street with a sequence of $360^{\circ}$ panoramic images which are captured with a Point Grey's Ladybug3 mounted on the top of Mitsubishi MMS-X 220 at $2 \mathrm{~m}$ intervals along the streets in urban environment (Chen et al., 2012), and projected/stitched on to one special 3D mesh called projection base plan (See Figure 1) which is equivalent to the DEM/DSM used to generate ortho-image with aerial/satellite images, and constructed with existing 2D/3D GIS data along the street. Onboard GPS/IMU, speedometer and post sequence image analysis technology such as bundle adjustment provided high accuracy position and attitude data for these panoramic images, and laser scanning data. This makes it possible to make large scale panoramic street strip image maps with characters of both traditional ortho-image and multi-perspective strip panorama for most types of cities. The purpose of this paper is to provide a kind of low-cost, shortcut and useful GIS data making method to meet the requirements of navigation, driving direction pre-visualizations and augmented reality as demonstrated in Google Earth or Microsoft Virtual Earth with our Mobile Mapping System (MMS). So, the paper is arranged as the following. The overview about panoramic street strip image map generation with a sequence of $360^{\circ}$ panoramic images is presented in the next section, this is followed by the data acquisition with our MMS. Some results obtained with the method of this paper will be provided, and in the final we will give the conclusion.

\section{PANO-STREET OVERVIEW}

The purpose and the theory of making panoramic street strip image map with ground street level panoramic images are similar to that of aerial ortho-images. Figure 2 illustrates the flow diagrams of Pano-Street generation. More detail descriptions about these diagrams will be presented in the following subsections.

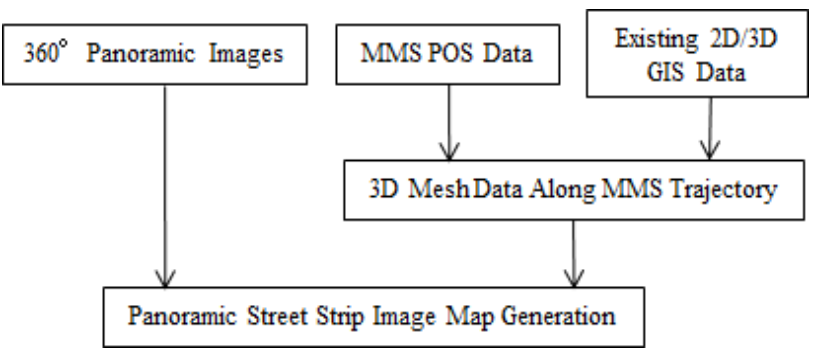

Figure 2. Flow diagrams of Pano-Street generation.

\section{1 $360^{\circ}$ Panoramic Images}

Panoramic images are a new type of visual data that provide many new possibilities as compared to the classic planar images since panoramic images allow observing a scene from various viewing directions. Large scale GIS for street-level viewing, geographical mapping and other location-based visualizations, high end security and surveillance, city planning, simulation and measurement analysis, entertainment solutions for lighting models, full dome projection content, tele-presence, virtual navigation and other immersive experiences are examples of such interesting applications. Other more applications of panoramic images for 3D close range photogrammetry have been also reported due to their abilities to record much visual information of the real environment in one image at field view angle of $360^{\circ}$ in longitude direction and $180^{\circ}$ in latitude direction, especially their usage in the narrow spatial sites. The most famous application example is that Google Street View has used panoramic cameras over the world to collect geo-referenced panoramic images of city environments to expand the Google web GIS database for more detail view of the ground surface.

The use of panoramic photographs dates back until the early years of photography. First panoramic images have been recorded with rotating frame cameras or by swing lens techniques. During the 19th century, panoramic cameras have been combined with angular reading in order to measure the rotation angle of the camera. Consequently, panoramic photography and the use of photo-theodolites were closely connected. These methods only create the analog panoramic photographs. The current digital panoramic images are mostly created by off-the-shelf stitching programs that can match uncalibrated frame images into a cylindric projection with limited user interactions or using spherical surface reflection mirror just for observing a scene of natural environments, e.g. for touristic purposes and low accuracy metric site documentation for facility management applications (Chapman \& Kotowski, 2000), or for the combination with 3D laserscanners. Some researchers (Amiri Parian \& Gruen, 2005; and Schneider \& Maas, 2005) have developed camera calibration approaches that, in addition to photogrammetric interior orientation parameters, model the specific rotating linescanning characteristics such as tumbling of the vertical 
rotation axis. A more detailed view on the history of panorama photogrammetry can be extracted from Luhmann (2005).

With the emergence of high resolution panoramic cameras such as Point Grey's Ladybug3 and Ladybug5, much more attentions of these will be put on their photogrammetric processes such as image matching, DEM and ortho-photo generation, aerial triangulation, map compilation, stereoscopic viewing, and 3D model texture mapping. This will cause another research and application great mass fervor in photogrammetry field (Chen et al., 2012).

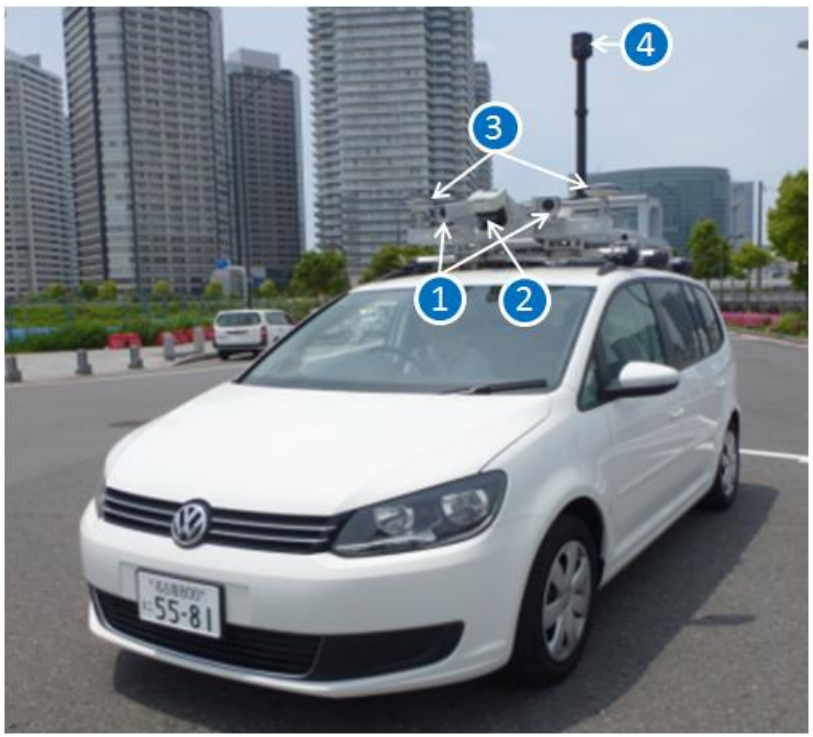

Figure 3. Overview of Mitsubishi MMS-X 220: (1) frame video cameras; (2)laser scanners; (3)GPS antennas; (4)Ladybug3 panorama camera.

In this paper one Point Grey Research, Inc.'s Ladybug 3 spherical camera mounted on the top of Mitsubishi MMS-X 220 (Figure 3) was used to collect the sequence of $360^{\circ}$ panoramic images at $2 \mathrm{~m}$ intervals along the streets. Ladybug3 is a multiple camera unit which is composed with six Sony CCD digital video cameras (See Figure 4a). Five of these cameras are arranged concentrically in a horizontal ring pointing outwards to produce a 360 degree panoramic image within the horizontal plane, with the sixth camera pointing vertically upwards. These cameras have a FireWire-B (IEEE1394-B) 800 Megabit interface and cabling to provide camera control and power and to implement video data transmission at the rate of 7 uncompressed frames per second and the rate of 15 compressed JPEG frames per second, each image being 1,600 x 1,200 pixels (2 Megapixels) in size (See Figure 4b). Each Ladybug3 camera unit can be supplied attached to a mast that can be mounted on a roof rack that has been placed on top of the mapping vehicle to provide a clear view of the surrounding objects. Since the camera's sensors have been accurately calibrated, it is possible to fuse the six images to form an almost complete spherical panorama. This panorama can therefore be considered to have been produced by a central projection camera that collects all light rays coming from all directions, incident on a point in space. The resulting two-dimensional plenoptic function can then be reprojected on any type of surface. We use the normal spherical surface as the projection of panoramic image (See Figure 4c).

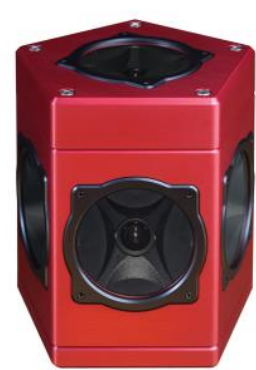

(a) Ladybug 3 body

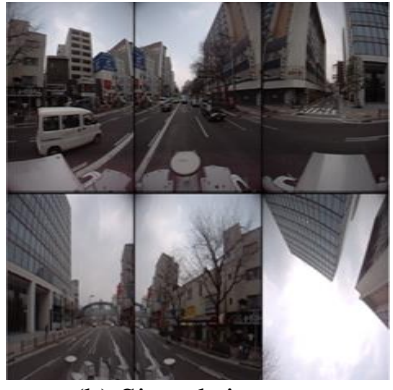

(b) Six sub-images

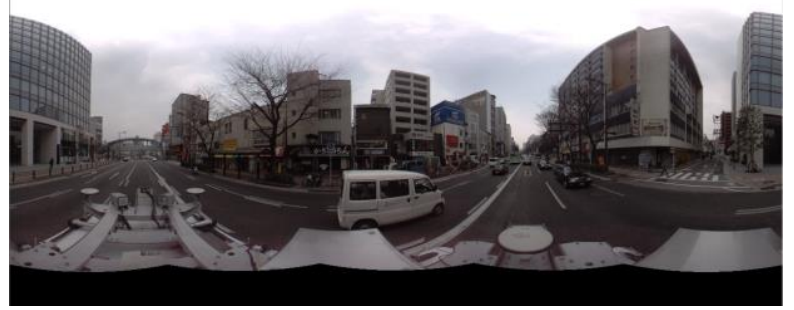

(c) $360^{\circ}$ Panoramic image

Figure 4. Ladybug3 and its six sub-images and the merged $360^{\circ}$ panoramic image.

\subsection{MMS POS Data}

The exterior orientation parameters (EOPs) of cameras are important to generate ortho-images or texture mapped $3 \mathrm{D}$ models with the traditional aerial or satellite images. These parameters could ensure these aerial or satellite images to be accurately projected on to the DEM/DSM and 3D models automatically. For our method of making panoramic street strip image map, the EOPs of each $360^{\circ}$ panoramic image obtained in last sub-section take the same roles as that of the traditional aerial or satellite images. Moreover, they are also important basis to construct the 3D mesh in Figure 1 along the moving route of the street.

The EOPs of all $360^{\circ}$ panoramic images are mainly provided by two methods: GPS/IMU integration and the traditional image based aerial triangulation, also called direct orientation method and indirect orientation method in modern photogrammetry. The direct orientation method provides the ability to directly relate the data collected by a remote sensing system to the Earth, by accurately measuring the geographic position and orientation of the sensor without the use of traditional ground-based measurements. Examples of where the direct orientation method are used in the airborne mapping industry include: scanning laser systems, Interferrometric Synthetic Aperture Radar systems (InSAR), the new state-ofthe-art digital line scanners systems such as the LH Systems ADS40, and more increasingly small format digital cameras and traditional film cameras. The direct orientation method is now also the main way used in all most MMSs to collect positioning and attitude information for the orientation of all sensors such as cameras, laser scanners, multi/hyper-spectral scanner, and Ground Penertrating Radar (GPR) etc. mounted on (Li, R., 1999; Tao, V., et al., 2007; Cazzaniga, N., et al., 2012). The indirect orientation method provides EOPs for images by aerial triangulation based on the object information and their corresponding image coordinates. As a result of using a mathematical model (collinearity equations) representing the transformation between the object and image spaces, the EOPs 
are determined, providing a relationship between the image coordinates and the global (or local) mapping coordinate. The combined bundle adjustment usually facilitates not only EOP determination, but may also involve rectification of the camera interior orientation parameters such as focal length, location of the principle point, coefficients of appropriate models representing lens distortion. Unfortunately, the significant part of the aerial triangulation cost is associated with the establishment of ground control points (GCPs), which might be prohibitive in case of mapping of remote areas. The indirect orientation method is only used in our MMS for system calibration and compensation of the errors in measurements obtained from GPS/IMU integration in urban areas where signal from satellites is often blocked by high buildings, trees and other objects. More detail will be described in other paper about the MMS indirect orientation method which is a combined aerial triangulation method with MMS $360^{\circ}$ panoramic images and the obtained GPS/IMU information as initial values of EOPs, only brief description about direct orientation method is introduced here.

The navigation component of our MMS provides position and orientation information for direction orientation method, and is composed with three roof-mounted GNSS receivers arranged in a triangular pattern, an IMU, and an odometer (Figure 3). The calculation of MMS position and orientation information is based on integration of data from the GNSS receivers, IMU, and odometer using standard GPS data processing software such as GrafNav. This is a little different from the POS data processing for airborne mapping systems where the GNSS receivers work well most within the platform moving time. The integration of GPS/IMU and odometer allows to achieve the required accuracy even in areas where the GNSS positioning alone is unreliable or entirely impossible. This happens mostly in urban areas where signal from satellites is often blocked by high buildings, trees and other objects.

The absolute system position calculated using the GNSS technology serves for compensating errors in measurements obtained from the inertial measurement unit. On the other hand, the relatively stable position (in a short-term scope) determined by IMU can be used to overcome areas where GPS fails. Additional information is received from odometers measuring speed and distance travelled depending on the rotation of wheels of the vehicle. There are two trajectory calculation methods that became widely used in practice, referred to mostly as loosely and tightly coupled. Both methods utilize Kalman's filters while when the loosely coupled method is applied, the path is pre-calculated using the GNSS differential method first. This path is then used when processing IMU data to update position and speed. When the tightly coupled method is used, the GNSS/INS data is processed simultaneously, making it possible to use at least two satellites for a phase update, which means a great advantage in difficult conditions with limited satellite signal reception.

Typically, GPS serves as the main positioning sensor by measuring ranges and range rates from a GPS receiver's antenna to the GPS satellites. Antenna-to-satellite intervisibility is a must. The accuracy degradation of the GPS solution is due to poor satellite spatial distribution, loss of lock, and satellite signal blockage. In addition, even by introducing multi-antenna configurations, the frequency of attitude determination may be too low (say, only $10 \mathrm{~Hz}$ ), which limits their use for some systems such as LIDAR (light detection and ranging) and other applications with expected high dynamics.

On the other hand, an INS acts as the main attitude sensor. The INS typically consists of three orthogonal accelerometers and three orthogonal gyroscopes. The accelerometer and gyroscope triads are parallel and establish the INS coordinate system. The three accelerometers sense the body-frame linear accelerations while the gyroscopes sense the body-frame angular velocities with minimum time delay. The estimation of the relative position and attitude is accomplished by integrating the sensed signals after being transformed to the appropriate computational coordinate frame.

For short time intervals, the integration of acceleration and angular rate results in extremely accurate velocity, position, and attitude with almost no noise or time lags. However, because the navigation information is obtained by integration, they exhibit low frequency drift. To obtain accurate navigation information over all frequencies, the INS navigation solution should be updated periodically using external measurements. For this purpose, GPS velocities and positions are used as update measurements, which complement the INS output in an ideal way.

Also, other navigational aids, such as odometers, inclinometers, or barometers, may provide additional observations for some subset of the navigational state. The successful use of an MMS critically depends on both the accuracy and the continuity of the navigation solution. A perfect MMS is, therefore, one that can operate without interruption in areas where a discontinuity in the data from one or more of the navigational sensors is expected.

\subsection{D Mesh Data Generation along MMS Trajectory}

To stitch all $360^{\circ}$ panoramic images captured with MMS into a long $360^{\circ}$ image strip along the city street, a kind of 3D mesh data illustrated in Figure 1, which represent the street scenes and act the similar role of the traditional DEM/DSM (Digital Elevation Model, Digital Surface Model) used to generate topdown ground ortho-image with airborne images and satellite images in photogrammetry, should be constructed and taken as the projection base plane of the panoramic street strip image map to be generated here.

The 3D mesh data may be a flat, curved, parametric, complex, or irregular surface. It may be parallel to the street, parallel to the associated path segment, and/or parallel to the direction of movement of the MMS as the $360^{\circ}$ panoramic images are collected. According to the different quality requirements for the panoramic street strip image map and the environmental conditions of the street, the 3D mesh data could be generated from laser scanned point cloud data, stereo-image matched point cloud, mono/stereo measured $2 \mathrm{D} / 3 \mathrm{D}$ vector data, existing 2D/3D GIS vector-based or raster-based data, or simply simulated regular or irregular tunnel-shaped (Figure 1a) or trough-shaped (Figure 1b) 3D model along MMS trajectory or city street center line by distance to the curb of the street or the front of the building facades on two sides of the street. Since the street scenes contains varying depths and many obstructions, it is not possible to always obtain perfect representation of the ground street scenes for 3D mesh data as the traditional DEM/DSM used to generate top-down ground ortho-image with airborne images and satellite images in photogrammetry even if the complete knowledge of the 
geometry and appearance of the scenes is available. Moreover, the MMS trajectory and its moving direction are always changeable to avoid obstacles such as the slow moving or parked vehicles or pedestrians, this also increases the uncertainty of the 3D mesh data created automatically along the MMS trajectory. The varying scales and resolutions on the $360^{\circ}$ ground panoramic images caused by the varying depths of street scenes also make it much more difficult to generate a homogeneous quality and seamless mosaiced panoramic street strip image than to generate top-down ground ortho-image with airborne images, satellite images, and the ground DEM/DSM in photogrammetry.

To simplify the creation of the 3D mesh data and increase the practicability of the generation of the panoramic street strip image map, an average depth for the street scenes and a 3D surface at the average depth illustrated in Figure 1 are determined based on the street center line, laser scanned point cloud data, the existing 2D/3D GIS vector-based or rasterbased data, and the MMS $360^{\circ}$ ground panoramic images. Figure 5 illustrates the section to create the 3D mesh data in the whole process for the panoramic street strip image map generation.

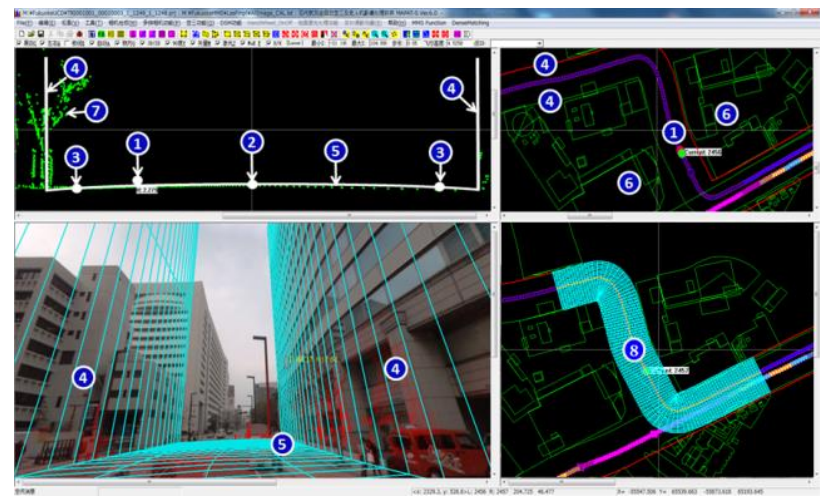

Figure 5. Interface of 3D mesh data generation for our MMS data processing system (left-up view: cross section of the 3D mesh data, which can be switched into stereo measurement mode with stereo $360^{\circ}$ panoramic images and aerial images; right-up view: MMS trajectory on 2D map, which can be switched into ground ortho-image and single aerial image mode; left-down view: 3D view for overlapped MMS laser point cloud and $360^{\circ}$ panoramic image; right-down view: hybrid measurement by laser point cloud and $360^{\circ}$ panoramic images), where the labelled number (1)denotes MMS position; (2)denotes the road center; (3)denotes road curbs; (4)denotes the sides of the 3D mesh data determined by the average distance;

(5)denotes the fitted road surface with laser point cloud; (6) denotes the building footprints on 2D map; (7)denotes the MMS laser point cloud; and(8)denotes the 3D mesh.

In Figure 5 a monitor snap shot view of our MMS data processing system is given to create a trough-shaped 3D mesh data illustrated in Figure 1b for panoramic street strip image generation along a street in accordance with the MMS laser scanned point cloud data, MMS trajectory data, the distances between the center line and the building facades of the both sides of the street, the existing 2D/3D GIS vector-based or raster-based data, the aerial images, and the MMS $360^{\circ}$ ground panoramic images in our hybrid photogrammetric workstation for processing the ground-borne mobile mapping system data and the airborne mapping system data. The trough-shaped 3D mesh data is composed with three parts: both street left and right sides which are determined by the average distances from the road center line to street scene objects such as buildings and trees etc., and the road surface cloud be fitted with MMS laser point by removing some obstacles such as parked or moving vehicles or pedestrians through $3 \mathrm{D}$ laser filtering methods or swiping the road surface with an approximate road section determined with the MMS height above the ground. All the three parts are extracted from one cross section illustrated on the left-up view in Figure 5 though the current MMS position by referencing the MMS $360^{\circ}$ ground panoramic image, laser point cloud and the existing 2D/3D GIS vectorbased or raster-based data. At certain interval such as the distance between two adjacent MMS positions, one cross section is formed and swept to the next section, and one through-shaped 3D mesh data could be obtained.

The right-down window in Figure 5 depicts the top view of the formed through-shaped 3D mesh data along the street. To smooth the obtained 3D mesh data, a spline function could be used to smooth the road street center line previously, then the road street center line is swept to both sides of street based on the road surface and the determined average distance to the street scene objects to define the bottom curbs of the 3D mesh. Based on the heights determined with laser point cloud, $360^{\circ}$ panoramic image and the existing 2D/3D GIS vector-based or raster-based data, the determined bottom curbs are swept up to form the two sides of the 3D mesh.

The tunnel-shaped 3D mesh data could be formed in the same manner by adding the top part for the above cross section to project some overpass such as high ways, galleries, and sheds etc., and tunnel objects on the panoramic street strip image map according to situation of the street scene and the resolution of the $360^{\circ}$ ground panoramic images. Figure 5 gives one sample of the tunnel-shaped 3D mesh data. In that case, the residential buildings are not tall, the street is not so wide, and some power wires and communication lines are overhead on the street. To incorporate all of the scene objects around the street, a top surface at the height of over the highest building along the street is constructed and combined with the two side surfaces and ground surface, and the final tunnelshaped 3D mesh data is formed for the panoramic street strip image map generation.

\subsection{Panoramic Street Strip Image Map Generation}

Top-down view orthoimage is one of the most fundamental products of photogrammetry, and is one of the most important and popular spatial data sources in modern GIS. With the development of imaging sensor, positioning devices, computer technology, and the image processing algorithms, especially the image matching algorithms, the generation of the ground orthoimage is becoming much more convenient, temporary, efficient, and economical since more precise and detail ground DEM/DSM could be quickly generated through parallel image matching with multi-view normal view and oblique view aerial images or high resolution satellite images whose spatial position and orientation are previously determined with GPS/IMU devices. However, the work is not so easy for the images taken on the ground, especially on the city street, because the more detail and precise DEM/DSM (i.e. 3D mesh data here) about a city street is difficult to be created due to the mutual occlusion of these street scene objects such as buildings, bushes, trees, transportation facilities, and other attachments of 
buildings and roads at different depths and heights along the street.

Different from the top-down view aerial images and satellite images, there are much more complex structures, textureless areas, frequent varying depths and scales, and occlusions in the ground $360^{\circ}$ panoramic images. To simplify the work of generating panoramic street strip image, the 3D mesh data has been simplified in last subsection and divided into different sections according to the ground $360^{\circ}$ panoramic images along the MMS trajectory (1) in Figure 5), the stitching process can start from the first captured ground $360^{\circ}$ panoramic image which corresponds to the first section of 3D mesh at the same distance interval as that of two neighbouring $360^{\circ}$ panoramic images along the MMS trajectory, and thus the steps to generate the panoramic street strip image could be taken as following.

Two side parts and the top part of the panoramic street strip image are firstly formed with the similar principle of aerial or satellite orthoimage generation by correlating the 3D mesh data and the ground $360^{\circ}$ panoramic images and their positions and orientations through the following photogrammetric collinear equation:

$$
\left[\begin{array}{c}
\mathrm{U} \\
\mathrm{V} \\
\mathrm{W}
\end{array}\right]=\lambda \bullet \mathrm{R}\left[\begin{array}{c}
\mathrm{X}-\mathrm{X}_{\mathrm{S}} \\
\mathrm{Y}-\mathrm{Y}_{\mathrm{S}} \\
\mathrm{Z}-\mathrm{Z}_{\mathrm{S}}
\end{array}\right]
$$

where, $(\mathrm{X}, \mathrm{Y}, \mathrm{Z})$ means the ground coordinates of one point on the 3D mesh, $(\mathrm{U}, \mathrm{V}, \mathrm{W})$ means the point's spherical coordinates on the corresponding ground $360^{\circ}$ panoramic image, $\lambda$ means the scale from $(\mathrm{X}, \mathrm{Y}, \mathrm{Z})$ to $(\mathrm{U}, \mathrm{V}, \mathrm{W}),\left(\mathrm{X}_{\mathrm{S}}, \mathrm{Y}_{\mathrm{S}}, \mathrm{Z}_{\mathrm{S}}\right)$ means the position of the image, and $\mathrm{R}$ means the rotation matrix constructed with the image's orientation angles. $(\mathrm{U}, \mathrm{V}, \mathrm{W})$ can be obtained through the following coordinate transformation from the $2 \mathrm{D}$ panoramic image plane coordinate $(\alpha, \beta)$ to the 3D sphere surface coordinate $(\mathrm{U}, \mathrm{V}, \mathrm{W})$ :

$$
\left[\begin{array}{c}
\mathrm{U} \\
\mathrm{V} \\
\mathrm{W}
\end{array}\right]=\left[\begin{array}{c}
\sin \beta \cos \alpha \\
\sin \beta \sin \alpha \\
\cos \beta
\end{array}\right]
$$

Since the ground $360^{\circ}$ panoramic images have certain ground overlaps, a portion of the $3 \mathrm{D}$ mesh may correspond to several ones among these ground $360^{\circ}$ panoramic images. An optimization sampling strategy could be taken to assign the color values for the pixel in the panoramic street strip image like the true or near true ground orthoimage generation with aerial images in photogrammetry. In that process, the direct above or nadir view aerial image is selected to remove the deformation caused by undulating ground and its center part is cut and as the main composition pasted on the ground orthoimage. The seam locations, occlusion parts, and leak portions would be mended with other direction view images.

For the ground $360^{\circ}$ panoramic images, the one corresponding to or roughly in front of the 3D mesh section depicted in Figure 5 is selected as the main composition pasted on the panoramic street strip image. This ensures the measurability of the panoramic street strip image, and makes it easy to remove the local deformed effect caused by perspective obstruction in the street scene, especially the object displacement on the seams of the panoramic street strip image.
The seam line process may be the key step in photogrammetric orthoimage generation and image mosaic in computer vision field. Although the multi-band fusion method (Burt and Adelson, 1983) is relatively mature and could provide perfect resolution for this problem, it is still computing cost. In our process, the weighted averaging of pixels in the ground $360^{\circ}$ panoramic image overlap regions is still used when the street scene become simple or in the regions where the 3D surfaces are flat. These regions are detected by multi-view image matching in our system and selected as the seamline areas between the neighbouring ground $360^{\circ}$ panoramic images. The final result we will obtain is a straight arranged panoramic image strip with correct topological relationship of ground scene objects along the trajectory of MMS, the parts on the image strip corresponding to the curved sections of the street may be covered by several ground $360^{\circ}$ panoramic images, and should be deformed and straighten, and the weighted averaging of pixels in the ground $360^{\circ}$ panoramic image overlap regions is needed.

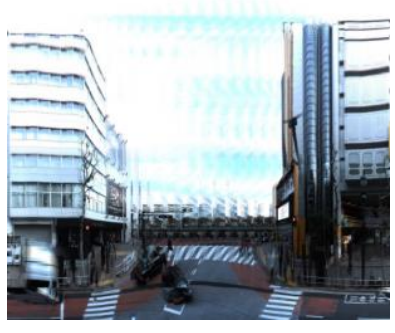

(a) Nearly ortho-stitched piece

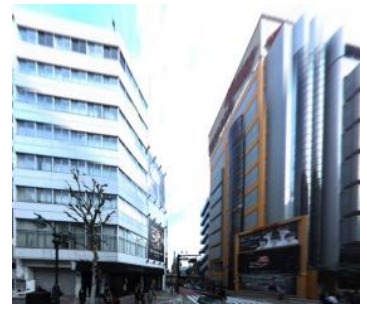

(b) The original image piece
Figure 6. The nearly ortho-stitched part for a piece of deep street scene far away from the 3D mesh and its corresponding part on the original perspective image.

Farther scenes away from the 3D mesh surface and the cross depth streets shown in Figure $6 \mathrm{a}$ will cause a repeated or stretched projection of same ground scene objects and blur where the far objects such as buildings wipe across several ground $360^{\circ}$ panoramic images when the MMS is moving along the street (Figure $6 \mathrm{~b}$ ). To overcome these phenomenon, only one single ground $360^{\circ}$ panoramic image near the center of the scene and the one just facing the depth of the cross street is selected and projected on a span area of several sections on the $3 \mathrm{D}$ mesh. The boundaries of the selected single ground $360^{\circ}$ panoramic image should be extended to some flat areas where the perspective distortions are not evident and the seamlines to the next section should not draw attention and could be easily connected as Figure 7.

After the two side parts and top part are formed for one $3 \mathrm{D}$ mesh section, the bottom part or the road surface part will be determined with either the forward sequential ground $360^{\circ}$ panoramic images or the backward sequential ones of the current ground $360^{\circ}$ panoramic image whose bottom part is not imaged and blanked. This causes the same section on the 3D mesh corresponding to the different ground $360^{\circ}$ panoramic images. To decrease the image displacement caused by different depths of ground scene objects, the nearest ground $360^{\circ}$ panoramic image to the current $3 \mathrm{D}$ mesh section is selected as the main composition pasted on the section part of the panoramic street strip image, the seam locations, occlusion parts, and leak portions caused by moving or parked vehicles or pedestrians would be mended with other more farther the 
forward sequential or backward sequential ground $360^{\circ}$ panoramic images.

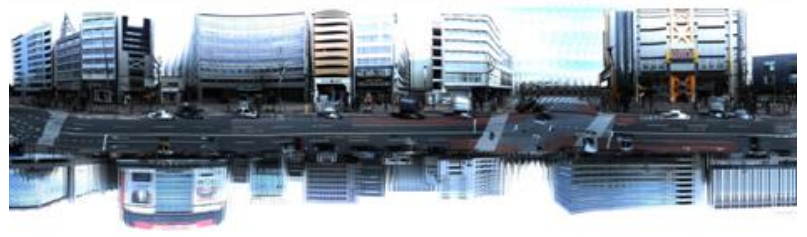

(a) Ortho-stitched "Pano-Street" piece for deep scene

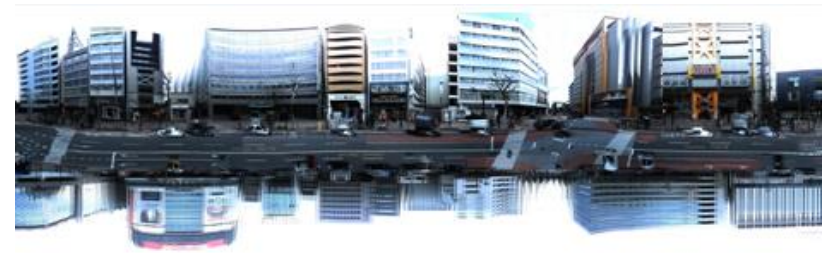

(b) Bridge jointed "Pano-Street" piece for deep scene

Figure 7. Contradistinction of the ortho-stitched piece and its bridge jointed piece for the deep scene.

In fact, all the parts of left and right, up and down sides for one section of the panoramic street strip image could be created through the image rectification and the image mosaic simultaneously in the aid of image matching to produce a gapless composite and fewer residual effects of moving vehicles or pedestrians with multi-direction overlapped ground $360^{\circ}$ panoramic images. The process is repeated from the first ground $360^{\circ}$ panoramic image on the start point of MMS trajectory to the last one on the end point of the trajectory, and a strip-shaped panoramic street image could be formed. Although this automatically composited panorama is often satisfactory, some visual artifacts such as color discontinuities, ghosting or blurring are still left over on the panoramic street image, we also allow for interactive refinement of the result. The user can paint rough strokes that indicate certain goals, such as the use of a certain viewpoint in a certain area of the panorama, such as local deformations for scenes at large dramatic varying depths.

\section{CONCLUSION}

In the last decades, much more efforts have been invested into large scale urban 3D model construction from vision-based 3D modelling and fusion 3D laser point clouds with images for these applications such as navigation, driving direction previsualizations and augmented reality as demonstrated in Google Earth or Microsoft Virtual Earth, but the progress on the street view level is not so amazing notable and the achieved results are still on the level of research. The reasons may be that the urban environments often lack textured areas, contain repetitive structures, many occlusions, strong lighting changes, and cast shadows. These properties make the above two methods difficult in the sense of finding enough reliable point matches between overlapping images and extracting key skeleton points from 3D laser point clouds for following 3D model construction. In fact, like aero ortho-images in GIS, a panoramic street strip image map is also a low-cost, shortcut and useful GIS data to meet the requirements of the above applications. It could be suitable for registration, transmission, visualization of street landscapes, and displayed on the internet to provide users a visual seamless immersive summary of a city.

This article explores a method of making panoramic street strip image map which is called as "Pano-Street" here and contains both sides, ground surface and overhead part of a street with a sequence of $360^{\circ}$ panoramic images which are captured with Point Grey's Ladybug3 mounted on the top of one Mitsubishi MMS-X 220 at $2 \mathrm{~m}$ intervals along the streets in urban environment. On-board GPS/IMU device, speedometer and post sequence image analysis technology such as bundle adjustment provided high accuracy position and attitude data for these panoramic images, and laser data. This makes it possible to make large scale panoramic street strip image maps for most types of cities. As the final result of this paper and a new derivative of MMS processed data, the panoramic street strip image map can be considered as a special 3D type strip map sharing a number of characteristic with $2 \mathrm{D}$ ortho-image, mainly the simple measurability, not only the heights of buildings, electric poles, trees, etc. on both sides of the street, but also the planimetric positions of some road ancillary facilities such as road curbs, manholes, and guard rails, could be measured on the image strip. Like the $2 \mathrm{D}$ ortho-image, the panoramic street strip image map is well suited for sequential route following, similar to following a travel narrative where the route to be taken is clearly line out. Like the panorama map, it can immerse the map reader in the landscape, giving a preview of what one may see when travelling along the depicted route. The panoramic strip map is therefore most suitable for information on landscape appearance before travel and for orientation during travel along a given route. It could also provide the basis for personalized documentation of a trip, and one optional map data source of street view for internet map service.

To create the panoramic street strip image map, the key step is to generate the 3D mesh data along the street. The quality and the precision of the 3D mesh will affect the geometric accuracy and the detail immersive nature of the panoramic street strip image map. It will be depended on the situation of the street scenes, the perceptual and geometric quality of the MMS processed data, and the requests of the users. Of course, the quality of the original ground $360^{\circ}$ panoramic images, such as their geometric resolutions and radiometric performance may be other important factor to create a satisfactory affect the panoramic street strip image map.

\section{REFERENCES}

Agarwala, A., Agarwala, M., Cohen, M., Salesin, D., and Szeliski, R., 2006. Photographing long scenes with multiviewpoint panoramas. ACM Transactions on Graphics 25, 3 (August), pp.853-861.

Amiri, J. and Gruen, A., 2005. A refined sensor model for panoramic cameras. International Archives of Photogrammetry, Remote Sensing and Spatial Information Sciences, Vol. XXXIV, Part 5/W16.

Baillard, C., Schmid, C., Zisserman, A., and Fitzgibbon, A., 1999. Automatic line matching and 3D reconstruction of buildings from multiple views. In Proc of ISPRS Conference on Automatic Extraction of GIS Objects from Digital Imagery, IAPRS, volume 32, pp.69-80. 
Bentrah, O., Paparoditis, N., and Pierrot-Deseilligny, M., 2004a. Stereopolis: An image based urban environments modeling system. In MMT 2004. The 4th International Symposium on Mobile Mapping Technology, Kunming, China.

Burt P.J. and Adelson E.H., 1983. A Multiresolution Spline with Application to Image Mosaics. ACM Trans. On Graphics, 2(4), pp.217-236.

Cazzaniga, N.E., Pagliari, D., Pinto, L., 2012. Photogrammetry for Mapping Underground Utility Lines with Ground Penertrating Radar in Urban Areas. International Archives of Photogrammetry, Remote Sensing and Spatial Information Sciences, Volume XXXIX-B1, 2012, XXII ISPRS Congress, 25 August - 01 September 2012, Melbourne, Australia, pp.297302.

Chapman, D., Kotowski, R., 2000. Methodology for the Construction of Large Image Archives of Complex Industrial Structures. Publikationen der DGPF, Band 8, Essen 1999.

Chen, T., Yamamoto, K., Chhatkuli, S., and Shimamura, H., 2012. Panoramic Epipolar Image Generation for Mobile Mapping System. International Archives of the Photogrammetry, Remote Sensing and Spatial Information Sciences, Volume XXXIX-B5, 2012, XXII ISPRS Congress, 25 August - 01 September 2012, Melbourne, Australia, pp.459464 .

El-Hakim, S., 2002. Semi-automatic 3d reconstruction of occluded and unmarked surfaces from widely separated views. In ISPRS Commission $V$ Symposium on Close Range Visualization Techniques, pp.143-148.

Frueh, C., Jain, S., and Zakhor, A., 2005. Data processing algorithms for generating textured $3 \mathrm{~d}$ building facade meshes from laser scans and camera images. Int. J. Comput. Vision, 61(2): pp.159-184.

Gupta, R., Hartley, R., 1997. Linear pushbroom cameras, IEEE PAMI, 19(9), pp.963-975.

Kopf, J., Chen, B., Szeliski, R., Cohen, M. 2010. Street Slide: Browsing Street Level Imagery. ACM Trans. Graph. 29, 4, Article 96(July 2010).

Li,R.X.,1999. Mobile Mapping- An Emerging Technology for Spatial Data Acquisition. The Ohio State University.

Luhmann, T. ,2005. A historical review on panorama imagery, International Archives of Photogrammetry, Remote Sensing and Spatial Information Sciences, Vol. XXXIV, Part 5/W16.

Rav-Acha, A., Engel, G., and Peleg, S. 2008. Minimal aspect distortion (MAD) mosaicing of long scenes. International Journal of Computer Vision 78, 2-3 (July), pp.187-206.

Román, A., and Lensch, H.P.A. 2006. Automatic multiperspective images. In Eurographics Symposium on Rendering, pp.83-92.

Román, A., Garg, G., and Levoy, M. 2004. Interactive design of multi-perspective images for visualizing urban landscapes. In IEEE Visualization 2004, pp.537-544.

Schneider, D., Maas, H.-G. ,2005. Application and accuracy potential of a strict geometric model for rotating line cameras. International Archives of Photogrammetry, Remote Sensing and Spatial Information Sciences, Vol. XXXIV, Part 5/W16.

Stamos, I. and Allen, P., 2000. 3d model construction using range and image data. In Computer Vision and Pattern Recognition, pp.531-536.

Surendra, P.S., Kamal, J., and Mandla, V.R., 2014. A New Approach Towards Image Based Virtual 3D City Modelling by Using Close Range Photogrammetry. ISPRS Annals of the Photogrammetry, Remote Sensing and Spatial Information Sciences, Volume II-5, ISPRS Technical Commission V Symposium, 23-25 June 2014, Riva del Garda, Italy, pp.328337.

Tao, V., Li, J., 2007. Advances in mobile mapping technology. ISPRS Book Series No. 4; Taylor \& Francis: London, UK, 176 pages.

Vincent, L. 2007. Taking online maps down to street level. Computer 40, 12 (December), pp.118-120.

Zhao, H. and Shibasaki, R., 2001. Reconstructing textured cad model of urban environment using vehicle-borne laser range scanners and line cameras. In ICVS '01: Proceedings of the Second International Workshop on Computer Vision Systems, pp.284-297.

Zheng, J., and Tsuji, S., 1992. Panoramic Representation for route recognition by a mobile robot, $I J C V, 9(1)$, pp.55-76.

Zheng, J. Y., Zhou, Y., Shi, M., 2004. Scene tunnels for seamless virtual tour, 12th ACM Multimedia, pp.448-451.

Zhu, Z., Hanson, A., Riseman, E. M., 2004. Generalized parallel-perspective Stereo Mosaics from Airborne Video. IEEE PAMI 26(2): pp.226-237. 